\title{
A Comparison between Divertor Heat Loads in ELMy and HRS H-Modes on J FT-2M
}

\author{
KAWASHIMA Hisato ${ }^{1)}$, UEHARA Kazuya ${ }^{1)}$, NISHINO Nobuhiro ${ }^{2)}$, KAMIYA Kensaku ${ }^{1)}$, \\ TSUZUKI Kazuhiro $^{1)}$, MOHAMMAD Bakhtiari ${ }^{1)}$, NAGASHIMA Yoshihiko ${ }^{3)}$, OGAWA Hiroaki ${ }^{1)}$, \\ HOSHINO Katsumichi ${ }^{1)}$, SUZUKI Sadaaki ${ }^{1)}$ and KUSAMA Yoshinori ${ }^{1)}$ \\ ${ }^{1)}$ Naka Fusion Research Establishment, Japan Atomic Energy Research Institute, Ibaraki 311-0193, Japan \\ ${ }^{2)}$ Hiroshima University, Hiroshima 739-8527, Japan \\ ${ }^{3)}$ National Institute for Fusion Science, Toki 509-8292, Japan \\ (Received 13 September 2004 / Accepted 16 October 2004)
}

\begin{abstract}
A comparison between divertor heat loads in ELMy and High Recycling Steady (HRS) H-modes was carried out by fast measurements of the two dimensional $D_{\alpha}$ emission, and the divertor electron temperature and ion saturation current on JFT-2M. The heat load for the HRS H-mode was maintained at the same level as the time-averaged heat load for the ELMy H-mode, indicating that the HRS H-mode has the attractive advantage of avoiding the severe ELM heat load. An enhancement of particle transport dominated the heat load for the HRS H-mode, whereas the large ELM heat load was induced by abrupt increases of both heat and particle transport.
\end{abstract}

Keywords:

2D $D_{\alpha}$ emission, divertor probes, ELMy H-mode, HRS H-mode, divertor heat load

Reduction of divertor heat load has been studied on JFT$2 \mathrm{M}[1,2]$. Recently, the HRS H-mode [3] was shown to strongly reduce the ELM activity, and thus to have potential for eliminating the severe ELM heat load on the divertor target. We compared the divertor heat loads between the ELMy H-mode and the HRS H-mode by fast measurements as follows.

In the JFT-2M tokamak $\left(R=1.31 \mathrm{~m}, a \sim 0.35 \mathrm{~m}, B_{\mathrm{T}} \leq\right.$ $2.2 \mathrm{~T}$ ), some unique electrostatic probes (divertor probes, ion sensitive probe, Mach probe, etc.) are installed to measure the electron/ion temperature, ion saturation current (equivalent to the particle flux), flow and so on, characterizing the scrapeoff layer (SOL) and divertor plasmas [1,4]. It has been possible to study the fast ( $<1 \mathrm{~ms}$ ) behavior of ELMs by sampling these probe signals at $1 \mathrm{MHz}$. A newly installed fast framing camera system (up to 40,500 frames/s), which tangentially views the lower divertor region as shown in Fig.1, measures the two dimensional (2D) $D_{\alpha}(656 \mathrm{~nm})$ emission profile [5]. In this paper, it is shown that the divertor plasmas for the ELMy and HRS H-modes are characterized by divertor recycling, and heat and particle fluxes from the fast camera and divertor probe measurements. Consequently, the difference of divertor heat loads between them is clarified for the first time.

Figures 2 and 3 show the time evolutions of ELMy and HRS H-mode discharges at the conditions of $I_{\mathrm{p}}=0.24-0.27$ MA, $B_{\mathrm{T}}=1.6 \mathrm{~T}, q_{95}=2.6-2.7, \bar{n}_{\mathrm{e}}=4-5 \times 10^{19} \mathrm{~m}^{-3}, D_{2}$ working gas and balanced NBI (1.1-1.4 MW) in the lower

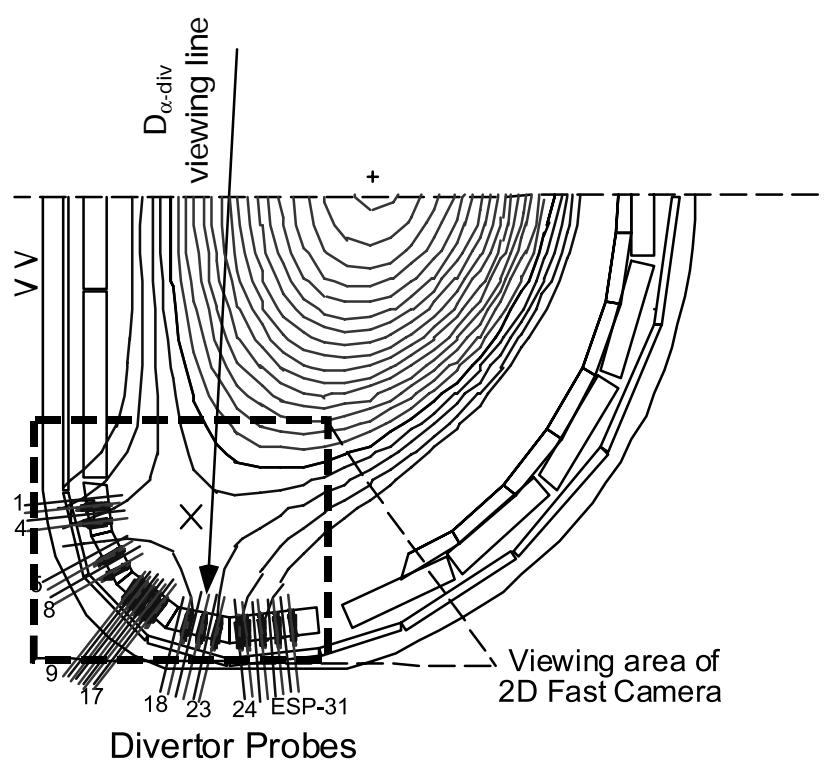

Fig.1 Viewing area of the 2D fast camera and locations of divertor probes on J FT-2M lower divertor region.

single null divertor configuration. An ELMy H-mode discharge was obtained under a low recycling condition just after the glow discharge cleaning (GDC). Relatively large amplitude ELMs appear after the ELM free H-mode, as shown in Fig.2(d), which is the line integrated $D_{\alpha}$ emission with a viewing line from the top port to the lower divertor (Fig.1). Figures 2(a)-(c) show images (turning to the left along 


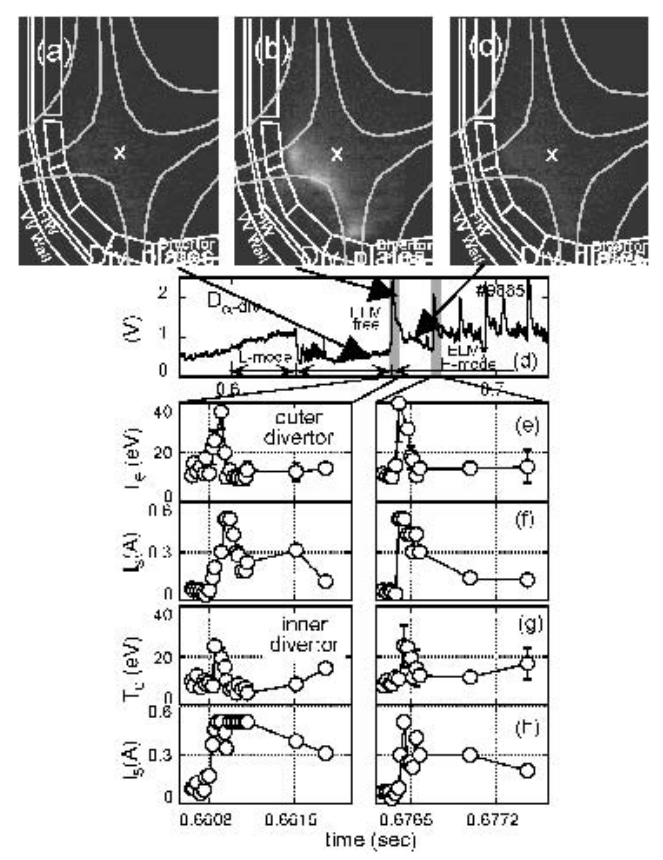

Fig. 2 Time evolution of the ELMy H-mode discharge.

the vacuum vessel toward to inner) of the $D_{\alpha}$ emission profile during the ELM free H-mode, an ELM event, and the profile after the event, respectively. These are extracted from the sequence of images (4,500 frames/sec) measured by the fast camera. The images show that the $D_{\alpha}$ emission was strongly intensified during the ELM event in front of both the inner and outer divertor targets: (b), in contrast with those for ELM free H-mode phase: (a) and the later relaxation phase: (c). Figures 2(e)-(h) show the time evolutions of $T_{\mathrm{e}}$ and $I_{\mathrm{s}}$ around the strike points on the outer and inner divertor targets at two time periods including ELMs. In these cases, the time resolution is restricted to $25 \mathrm{~ms}$ depending on the limit of bias sweep time, but it can still describe the time evolution in response to the ELM pulse as shown in the figures. During the ELM event, $T_{\mathrm{e}}$ and $I_{\mathrm{s}}$ increase respectively by $2 \sim 4$ and $5 \sim 6$ times compared with those just before its generation. These results show that large heat and particle fluxes arrive abruptly on the divertor targets along the field line during an ELM, increasing the recycling in front of the divertor targets. On the other hand, a typical HRS H-mode was obtained on the 6th discharge after GDC with a high recycling condition (Fig.3). The HRS transition occurs at $t \sim 0.645 \mathrm{sec}$ after a brief ELM-free phase with enhanced $D_{\alpha}$ emission, which is rather uniformly spread in space (Fig.3 (a)-(c)), rather than being localized as it is during an ELM event. $T_{\mathrm{e}}$ remains at a low level ( $10 \mathrm{eV})$ (Fig.3 (e), (g)), whereas $I_{\mathrm{s}}$ increases in a manner similar to the $D_{\alpha}$ enhancement (Fig.3 (d), (f), (h)). These findings show that the heat load for the HRS H-mode is induced dominantly by an enhancement of particle transport.

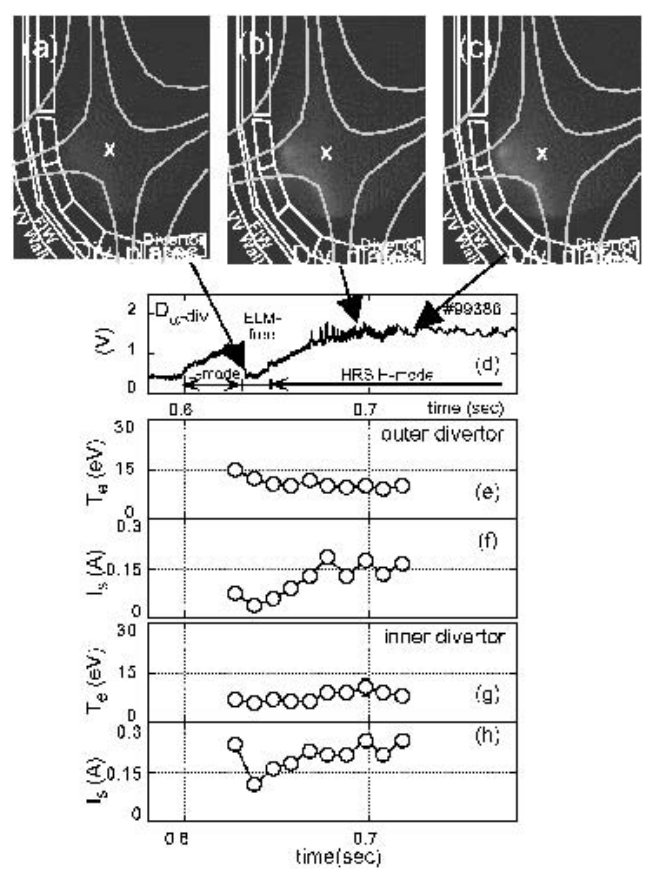

Fig. 3 Time evolution of the HRS H-mode discharge.

For the ELMy and HRS H-mode, the heat load $\left(\Gamma_{\text {heat }}\right)$ on the divertor target is evaluated from $\gamma J_{\mathrm{s}} T_{\mathrm{e}}$, where $J_{\mathrm{s}}$ is the ion saturation current density. The heat transmission coefficient $\gamma$ is assumed to have a value of 7 [6]. It may change depending on the recycling conditions and NBI power [7], but we expect that its change in these experiments is small due to the relatively low NBI power. As a result, $\Gamma_{\text {heat }}$ at the HRS steady phase becomes $\sim 0.3 \mathrm{MW} / \mathrm{m}^{2}$ around the strike points on both the outer and inner divertor targets. It corresponds approximately to the time-averaged heat load for the ELMy $\mathrm{H}$-mode and $\sim 15 \%$ below the peak value during an ELM event.

Thus, we can obtain the highly resolved time evolutions of divertor recycling, and heat and particles fluxes during the fast ELM event, and have demonstrated an attractive feature of the HRS H-mode regarding the reduced peak heat load through these measurements.

The authors would like to thank the JFT-2M team for their support and fruitful discussions.

[1] H. Kawashima et al., Nucl. Fusion 41, 257 (2001).

[2] H. Kawashima et al., J. Nucl. Matter 313-316, 1338 (2003).

[3] K. Kamiya et al., Nucl Fusion 43, 1214 (2003).

[4] K. Uehara et al., Jpn. J. Appl. Phys. 42, 657 (2003).

[5] H. Kawashima et al., Bul. J. Plasma Fusion 28aB12P (2003), p.242.

[6] P.C. Stangeby et al., Nucl. Fusion 30, 12 (1990).

[7] N. Asakura et al., Nucl. Fusion 35, 381 (1995). 